\title{
Evaluación de las pruebas motrices relacionadas con el lanzamiento en deportistas con discapacidad intelectual Evaluation of the motor shooting tests in athletes with intellectual disabilities
}

\author{
Jorge Abellán Hernández \\ Universidad de Castilla La Mancha
}

\begin{abstract}
Resumen: Las características de las personas con discapacidad intelectual incluyen un bajo desarrollo de las habilidades y destrezas básicas y de la coordinación. Sin embargo apenas existen estudios que se centren en investigar su desarrollo o a comparar diferentes niveles o grupos de prácticas en pruebas relacionadas con el deporte. El objetivo principal de esta investigación ha sido estudiar el rendimiento de deportistas con discapacidad intelectual en tres pruebas motrices. Además se compararon los resultados en base a dos grupos de análisis creados en función de su rendimiento en el lanzamiento a portería. Los resultados muestran que los participantes exitosos en el lanzamiento a portería también obtienen mejores resultados en el lanzamiento de precisión y en el total de las pruebas evaluadas. Parece que los participantes exitosos tienen un desarrollo integral de la habilidad de lanzar y son capaces de demostrarla en diferentes situaciones.
\end{abstract}

Palabras clave: pruebas motrices, deporte adaptado, discapacidad intelectual.

Abstract: People with intellectual disabilities present a low development of their coordination skills. However there are not many studies focused in its development or in compare different levels or groups of practice in specific sport tests. The main objective of this paper is to study the performance of athletes with intellectual disabilities in three different motor tests. Also results in base of two different groups (successful and less successful in the shooting test) were compared. Results showed that the successful group in the shooting test obtained better results in the accuracy test and the total score too. It seems that successful group had a total development of the shooting skill and they are able to show it in different situations.

Key words: motor tests, adapted sport, intellectual disabilities.

\section{Introducción}

La actividad física adaptada y el deporte adaptado son disciplinas en desarrollo que se están implantando gradualmente en nuestra sociedad (Pérez, 2009). Sin embargo, dentro de las disciplinas de deporte adaptado, las practicadas por las personas con discapacidad intelectual (D.I.) se están quedando atrás en comparación con el resto de deportes adaptados para personas con otras discapacidades (físicas y sensoriales fundamentalmente). El desarrollo más pobre del deporte para personas con D.I. parece tener una causa histórica, como indican las revisiones de los juegos paralímpicos (Hernández Vázquez, 2000; Mendoza, 2009) en los que el deporte para personas con discapacidad intelectual ha sido el último en incorporarse. Además la existencia de múltiples clasificaciones de las personas con D.I. no ayudan, dando lugar a la aparición de cierto caos terminológico (Mendoza, 2009).

La D.I. está referida a las limitaciones significativas que posee el individuo en el plano cognitivo, intelectual y de conducta adaptativa y que aparecen antes de los 18 años (AAIDD, 2010; Verdugo, 2003). Las personas con D.I. reflejan una baja competencia motriz (Molina \& Beltrán, 2007) entendida como la incapacidad de superar una determinada situación o problema motriz (Ruiz, 1995). Además suelen tener problemas de coordinación (Molina \& Beltrán, 2007).

Por lo tanto las personas con D.I. tienen un desarrollo dispar de sus habilidades y destrezas básicas. Sin embargo no existen muchos estudios previos que evalúen las capacidades motrices y coordinativas de estas personas cuando practican deporte. En la investigación realizada por Lejcarova (2009) se analizó la coordinación de niños de 9 a 11 años en función de su nivel de D.I. Los resultados muestran que el rendimiento en las pruebas motrices utilizadas disminuye con el aumento del grado de D.I. de los participantes evaluados.

En este sentido existen más estudios centrados en analizar las capacidades coordinativas de las personas con D.I. Carmeli et al. (2008) comprobaron que las personas con DI obtuvieron una puntuación significativamente más baja en un conjunto de pruebas sensoriomotoras. También se han medido los efectos de un entrenamiento en el estado

Fecha recepción: 25-07-12- Fecha envío revisores: 26-07-12- Fecha de aceptación: 21-12-12 Correspondencia: Jorge Abellán Hernández

Facultad de Educación Universidad de Castlla La Mancha

Plaza de la Universidad 302071 Albacete (España)

E-mail: jorge.abellan@uclm.es físico y las habilidades motrices de adultos jóvenes con D.I. y diagnosticados con autismo (Hayakawa \& Kobayashi, 2011). Después de tres meses de entrenamiento (1 día a la semana durante 30 minutos) utilizando máquinas construidas por uno de los autores del estudio, se observo que los participantes mejoraron de forma significativa en carrera de $50 \mathrm{~m}$., tiempo medio en desplazamientos de $10 \mathrm{~m}$. y en $10 \mathrm{~m}$. caminando entre obstáculos. Sin embargo ninguna de las pruebas motrices utilizadas en estas investigaciones son destrezas específicas de uno o varios deportes y se centran más en habilidades y destrezas básicas y en el uso de baterías y pruebas estandarizadas.

En cuanto al desarrollo de investigaciones específicas de pruebas o test motores para personas con D.I. relacionadas con el deporte destaca la investigación de Van Biensen et al. (2010). En ella se comparo a jugadores de tenis de mesa élite con D.I. y sin D.I. en un test estandarizado en el que se analizaba su resto. Los deportistas con D.I. obtuvieron significativamente menos aciertos al tratar de adaptar sus restos a la velocidad de la bola y además tuvieron un tiempo de reacción más lento que sus compañeros sin D.I.

La presente investigación se ha posicionado cerca del mundo del deporte adaptado, desechando las pruebas motrices estandarizadas y utilizando pruebas motrices deportivas. Estas pruebas no disponen de la validez científica de las baterías motrices, sin embargo proporcionan información más cercana a la realidad deportiva, objetivo de esta investigación. Además son pruebas con mucha tradición en el mundo del deporte adaptado. En esta investigación participan únicamente deportistas con D.I. pertenecientes al nivel deportivo G3 (para más detalles ir a la sección de participantes). Debido a sus características, estos deportistas no pueden realizar el deporte en su totalidad, por lo que realizan pruebas motrices. Estas pruebas se corresponden con habilidades o destrezas básicas aplicadas a diferentes deportes. En concreto se utiliza la habilidad del lanzamiento, en tres tipos distintos de contextos correspondientes con tres deportes. El lanzamiento a portería es una prueba motriz extraída del fútbol sala, el lanzamiento de a canasta es una prueba motriz extraída del baloncesto, mientras que el lanzamiento de precisión es una prueba motriz que se corresponde con el atletismo en su disciplina de lanzamientos.

Desdelapsicología del deportesehavenidoutilizando la comparación entre expertos y novatos como forma de analizar las diferencias en comportamiento motor y en rendimiento de deportistas de diferente nivel de pericia (French \& McPherson, 2004). Sin embargo cuando se quieren comparar los comportamientos de deportistas en acciones muy 
concretas parece más adecuado realizar una clasificación en base a su rendimiento en una determinada acción (Whiting, 1986). Por ejemplo Savelsbergh, Van der Kamp, Williams y Ward (2005) dividieron a porteros expertos en dos grupos, exitosos y menos exitosos en base a su porcentaje de aciertos parando penaltis, para posteriormente buscar diferencias en su anticipación y en su comportamiento visual. En esta investigación parece más adecuado utilizar este último criterio por dos motivos, en primer lugar las tareas que se miden no corresponden con acciones globales de ningún deporte, por lo que son muy específicas, de manera que un determinado deportista considerado experto puede no dominar esta faceta del deporte y en segundo lugar debido a las características específicas de la muestra. Todos los participantes de esta investigación tienen D.I. y pertenecen al mismo grupo de práctica por lo que clasificarlos en función de su actuación en una determinada prueba motriz (lanzamiento a portería) nos parece la opción más adecuada.

El principal objetivo de este estudio es examinar las diferencias en el rendimiento en tres pruebas motrices distintas de deportistas con D.I. Adicionalmente se estudia la relación entre su acierto en el lanzamiento a portería y su acierto en las otras dos pruebas motrices, para ello se crearon dos grupos distintos de análisis, deportistas exitosos y menos exitosos en función de la puntuación obtenida en la prueba de lanzamiento a portería.

\section{Metodología}

\section{Participantes}

Un total 29 deportistas con D.I. (33.59 \pm 13.8 años) tomaron parte en el estudio. Todos los participantes (18 hombres y 11 mujeres) pertenecían a 8clubes dela Federación de Castilla-La Mancha de deportes para personas con discapacidad intelectual (FECAM). Tras la realización de las pruebas los participantes fueron clasificados en dos grupos, exitosos y menos exitosos en base a su puntuación en el lanzamiento a portería (para más detalles ir a la sección de resultados).

Todos los participantes competían en categoría G3 de FECAM, que establece los siguientes requisitos para participar en ella:

«Está dirigido a aquellos deportistas que por sus competencias motrices necesitan una adaptación rigurosa del reglamento o necesitan realizar pruebas motrices (individuales o colectivas) de la modalidad deportiva correspondiente adaptadas a sus posibilidades.

Las características de los deportistas participantes en este nivel son:

-Necesita ayuda verbal de un monitor o voluntario.

-Movimiento escaso y poca fuerza funcional en todas las extremidades.

-Dificultad significativa para comprender y llevar a cabo consignas verbales.

-Depende de ayudas técnicas para su movilidad (distintos tipos de sillas de ruedas, muletas, bipedestadores, andadores)» (FECAM, 2012a:4)

\section{Materiales}

Los materiales dependen de cada una de las tres pruebas motrices realizadas (para más detalles ver la sección procedimiento). A continuación se enumeran todos los materiales utilizados, divididos en pruebas:

-Lanzamiento a canasta: balón de gomaespuma y canasta de $1 \mathrm{~m}$. de diámetro x $1 \mathrm{~m}$. de altura.

·Lanzamiento de precisión: 3 aros (de 80, 60 y 40 cm. de diámetro respectivamente), soportes para aros y pelotas de tenis.

-Lanzamiento a portería: 1 portería de fútbol sala, balones de gomaespuma y la figura de un portero de plástico.

\section{Procedimiento}

Todos los participantes completaron tres pruebas motrices. Las pruebas motrices son una de las disciplinas clásicas en las competiciones de deportes para personas con discapacidad intelectual y cuentan con gran tradición tanto en competiciones internacionales, nacionales y autonómicas. El desarrollo de las pruebas se explica a continuación (FECAM, 2012b):

-Lanzamiento a canasta: El deportista realiza 5 lanzamientos a canasta, 1 desde una distancia de $1 \mathrm{~m}$. y 2 desde las distancias de 2 y 3 m. (Figura 1). Se puntúa con 1 punto si consigue anotar desde la distancia de $1 \mathrm{~m}$. y 2 puntos por cada canasta anotada desde 2 y $3 \mathrm{~m}$. Si el deportista intenta lanzar los 5 tiros a canasta recibe 1 punto (aunque no anote). Si no realiza la prueba recibe 0 puntos.

-Lanzamiento de precisión: El deportista realiza 7 lanzamientos de precisión desde una línea a una distancia de $2 \mathrm{~m}$. del objetivo. 3 lanzamientos al aro más grande ( $80 \mathrm{~cm}$.), 2 lanzamientos al aro mediano (60 cm.) y 2 lanzamientos al aro pequeño $(40 \mathrm{~cm}$.) (Figura 2). Por cada lanzamiento que atraviese el aro grande se obtendrá 1 punto, por cada lanzamiento que atraviese el aro mediano obtendrá 1 punto y por cada lanzamiento que atraviese el aro pequeño obtendrá 2 puntos. Por realizar la prueba recibirá 1 punto más (aunque no anote) y si no realiza la prueba recibe 0 puntos.

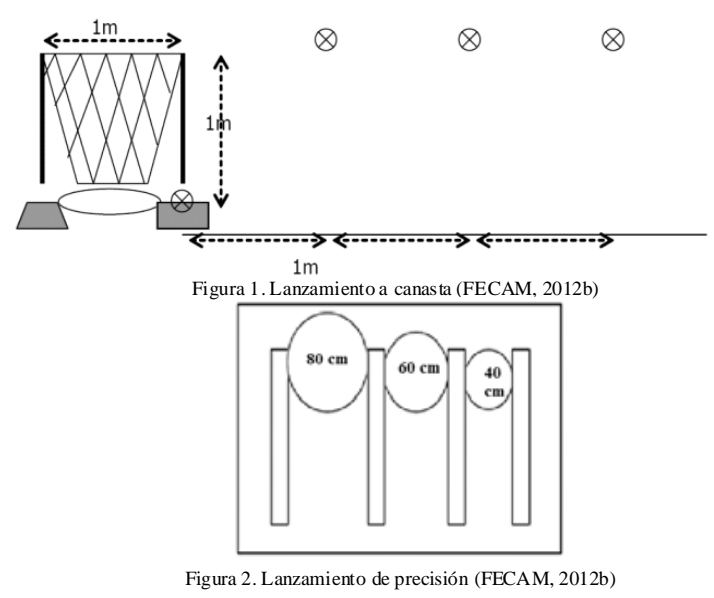

-Lanzamiento a portería: el deportista realiza 5 lanzamientos a portería con el pie (uno de los deportistas realizó los lanzamientos a portería con la mano, ya que se desplazaba en silla de ruedas, dicho participante fue encuadrado en el grupo de exitosos debido a su puntuación). Se realiza un lanzamiento desde cada una de las 5 zonas (Figura 3). Si el deportista anota el lanzamiento desde la zona 1 recibe 1 punto. Si anota lanzamientos desde las otras cuatro zonas (2, 3, 4 y 5) recibe 2 puntos. Si realiza la prueba obtiene 1 punto (aunque no anote ningún lanzamiento) y si no realiza la prueba recibe 0 puntos.

\section{Variables dependientes}

Las variables dependientes analizadas se refieren al rendimiento de cada uno de los participantes en las pruebas motrices realizadas. A continuación se enumeran dichas variables dependientes:

-Portería: puntuación obtenida en la prueba de lanzamiento a portería. La puntuación máxima que se puede obtener en esta prueba es 10.

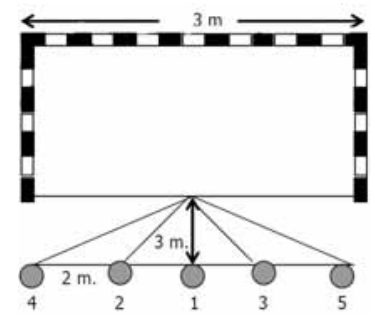

Figura 3. Lanzamiento a portería (FECAM, 2012b)

-Precisión: puntuación obtenida en la prueba de lanzamiento de precisión. La puntuación máxima que se puede obtener es de 10 puntos.

-Canasta: puntuación obtenida en la prueba de lanzamiento a canasta. La puntuación máxima que se puede obtener en esta prueba es de 10 puntos. 
-Total: suma de las puntuaciones obtenidas en las tres pruebas anteriores. La puntuación máxima es de 30 puntos.

Para cada variable dependiente se realizó una prueba T de Student con el objetivo de comparar los resultados en rendimiento de los dos grupos creados (exitosos vs. menos exitosos).

\section{Resultados}

Los 29 participantes han sido ordenados de mayor a menor, en base a la puntuación que obtuvieron en la prueba de lanzamiento a portería. A partir de esta clasificación se han formado tres grupos: exitosos, intermedios y menos exitosos. Las puntuaciones de todos los participantes en las distintas pruebas motrices así como en el total de la puntuación se muestran en la Tabla 1.

La prueba T de Student revela diferencias significativas entre los grupos creados en la prueba de lanzamiento a portería $(\mathrm{t}(15)=3.09$, $p=.007)$. Como es obvio debido a la forma de crear los grupos de análisis, los deportistas exitosos obtuvieron significativamente más puntuación en la prueba de lanzamiento a portería (8.86) que los menos exitosos (5.8).

Además el grupo exitosos obtuvo más puntuación media en la prueba de precisión (7.86) que el grupo menos exitoso (6), el grupo exitoso consiguió un rendimiento significativamente mejor $(\mathrm{t}(15)=3.05$, $p=.008)$.

Finalmente se encuentran diferencias significativas en las puntuaciones totales entre los dos grupos $(\mathrm{t}(15)=3.95, p=.001)$. Los deportistas exitosos fueron mejores en la suma de las tres pruebas motrices, con una puntuación media (23.86) más alta que la de sus compañeros menos exitosos (16.9).

Tabla 1. Clasificación de los participantes en base a su rendimiento en la prueba delanzamiento a porteŕa.

\begin{tabular}{cccccc}
\hline Ranking & Portería & Precisión & Canasta & Total & Grupo \\
\hline $1^{\circ}$ & 10 & 10 & 8 & 28 & Exitoso \\
& 10 & 8 & 8 & 26 & Exitoso \\
& 10 & 8 & 7 & 25 & Exitoso \\
& 10 & 8 & 6 & 24 & Exitoso \\
& 10 & 4 & 8 & 22 & Exitoso \\
$2^{\circ}$ & 10 & 2 & 8 & 20 & Exitoso \\
$3^{\circ}$ & 9 & 5 & 8 & 22 & Exitoso \\
& 8 & 6 & 10 & 24 & Intermedio \\
& 8 & 6 & 10 & 24 & Intermedio \\
& 8 & 10 & 5 & 23 & Intermedio \\
& 8 & 6 & 8 & 22 & Intermedio \\
& 8 & 6 & 6 & 20 & Intermedio \\
& 8 & 6 & 6 & 20 & Intermedio \\
$4^{\circ}$ & 8 & 6 & 5 & 19 & Intermedio \\
& 8 & 4 & 5 & 17 & Intermedio \\
$5^{\circ}$ & 7 & 2 & 6 & 16 & Intermedio \\
& 7 & 6 & 6 & 19 & Intermedio \\
& 7 & 6 & 6 & 19 & Intermedio \\
$6^{\circ}$ & 6 & 3 & 5 & 15 & Intermedio \\
$7^{\circ}$ & 6 & 8 & 8 & 22 & Menos exitoso \\
& 6 & 6 & 6 & 20 & Menos exitoso \\
& 6 & 6 & 6 & 18 & Menos exitoso \\
& 5 & 1 & 4 & 16 & Menos exitoso \\
$8^{\circ}$ & 4 & 6 & 10 & 11 & Menos exitoso \\
& 4 & 6 & 8 & 20 & Menos exitoso \\
& 4 & 8 & 6 & 18 & Menos exitoso \\
& 4 & 6 & 7 & 17 & Menos exitoso \\
& 3 & 2 & 4 & 9 & Menos exitoso \\
\end{tabular}

\section{Discusión}

El principal objetivo de esta investigación era estudiar las diferencias en el rendimiento obtenido por deportistas con D.I. en tres pruebas motrices distintas. En base a los resultados obtenidos en el lanzamiento a portería se crearon dos grupos, deportistas exitosos y menos exitosos. Los deportistas exitosos fueron significativamente mejores en las prueba de precisión y en el total de las tres pruebas realizadas, además de obtener significativamente más puntuación en la prueba de lanzamiento a portería, lo que era previsible debido a la forma en la que se crearon los grupos de análisis. Sin embargo los deportistas exitosos no fueron significativamente mejores en el lanzamiento a canasta, aunque si consiguieron una media de puntos superior a los menos exitosos.

Las pruebas motrices se presentan como una alternativa a los deportes adaptados para aquellos deportistas cuya D.I. les dificulta la toma de decisiones

El grupo de deportistas exitosos estaba compuesto por participantes con una puntuación en el lanzamiento a portería a partir de 9 (>9) y el grupo de deportistas menos exitosos estaba compuesto participantes con una puntuación en el lanzamiento a portería de 6 o menos $(\mathrm{d}<6)$. Los participantes con una puntuación entre los dos grupos (rango 7-8) se consideraron grupo intermedio. El resultado de esta distribución es el de siete participantes exitosos (34.14 \pm 12.21 años), doce participantes intermedios (30.58 \pm 11.93 años) y diez participantes menos exitosos (36.8 \pm 17.17 años). Debido al objetivo del estudio el grupo intermedio fue excluido del siguiente análisis. Los dos grupos restantes (exitosos y menos exitosos) se utilizaron para análisis posteriores.

Las puntuaciones medias de en las pruebas motrices, divididas en función de los grupos creados se presentan en la Figura 4.

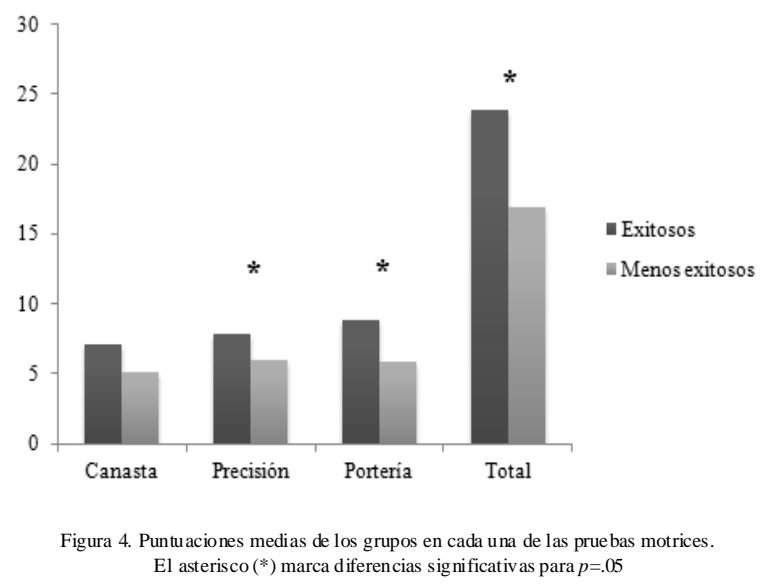

en contextos deportivos (nivel G3), por tanto en estas pruebas se presentan actividades centradas únicamente en la ejecución motriz de determinadas tareas. Deesta manera esta investigación se aleja de estudios previos centrados tan solo en el rendimiento de test motores estandarizados y alejados del mundo del deporte. En los resultados se muestra que los deportistas más exitosos en el lanzamiento a portería también lo son en el lanzamiento de precisión y en el total de las tres pruebas motrices evaluadas, por lo que aunque las personas con D.I. presentan problemas en el desarrollo de sus habilidades y destrezas básicas, entre ellas el lanzamiento y por lo tanto problemas en la coordinación (Molina y Beltrán, 2007) se demuestra que los que tienen un dominio superior de la destreza del lanzamiento son capaces de demostrarla en diferentes situaciones de evaluación. Estos resultados están de acuerdo con los estudios previos en deportistas sin discapacidad, en los que los pertenecientes a grupos más exitosos y expertos eran capaces de obtener mejores resultados en más de una faceta del juego (Williams, 2000).

Es interesante destacar el hecho de que los lanzamientos a portería se realizaron con los pies, mientras que los lanzamientos de precisión y los lanzamientos a canasta se realizaron con las manos, por lo que el superior rendimiento de los deportistas más exitosos en las pruebas de lanzamiento a portería y lanzamiento de precisión delata un desarrollo integral de la habilidad de lanzamiento por parte de los deportistas, es decir los deportistas del grupo más exitoso eran capaces de ser más precisos en sus lanzamientos tanto con las manos como con los pies.

En los estudios previos se ha comparado a las personas con D.I. en tests motores estandarizados (Carmeli, et al., 2008; Lejcarova, 2009) o en pruebas deportivas (Van Biensen, et al., 2010) con personas $\sin$ D.I. En esta investigación se presenta una nueva perspectiva de trabajo, en el que se compara a deportistas con D.I. del mismo nivel de práctica 
(G3) en función de su rendimiento. Por lo tanto esta investigación abre una nueva vía de trabajo en el deporte adaptado, con implicaciones en el diseño y evaluación del entrenamiento de los deportistas con D.I., además de con posibles implicaciones en el desarrollo denuevas pruebas motrices cercanas a la práctica deportiva real.

En conclusión, los deportistas más exitosos presentan un desarrollo integral de la habilidad de lanzar y por tanto lo pueden demostrar en diferentes situaciones de análisis.

\section{Agradecimientos}

La toma de datos de esta investigación se realizó durante el II Campeonato Regional de Pruebas Motrices celebrado en Toledo en Noviembre de 2011, organizado por la Federación de deportes para personas con discapacidad intelectual de Castilla-La Mancha (FECAM) con el apoyo de la Fundación Mapfre a través de su programa «Juntos lo logramos».

\section{Referencias}

Asociación americana de discapacidad intelectual y del desarrollo (AAIDD) (2010). Definition of intelectual disability. Disponible en www.aamr.org

Carmeli, E., Bar-Yossef, T., Ariav, C., Paz, R., Sabbag, H., \& Levy, R. (2008). Sensorimotor impairments and strategies in adults with intellectual disabilities. Motor Control, 12, 348-361.

Federación de Castilla La Mancha para personas con discapacidad intelectual (FECAM) (2012a). Normativa 2012. Disponible en www.fecam.ws

Federación de Castilla La Mancha para personas con discapacidad intelectual (FECAM) (2012b). Batería pruebas motrices. Disponible en www.fecam

French, K., \& McPherson, S.L. (2004). Development of Expertise in Sport. En Weiss, L.R. (Ed.). Development Sport and Exercise Psychology (pp. 403-423). Virgantown: Fitness Inovation Technology.
Hernández Vázquez, F. (2000). La educación física, el deporte y la diversidad en Secundaria. Apunts: Educación Físicay Deportes, 60, 6-12.

Lejcarova, A. (2009). Coordination skills in 9 to 11 years old pupils at practical elementary schools in relationship to their degree of intellectual disability. Acta Universitatis Palackianae Olomuensis Grymnica, 39(4), 53-62.

Mendoza, N.(2009). Propuestas prácticas de Educación Física inclusiva para la etapa de secundaria. Barcelona: INDE.

Molina, J. P., \& Beltrán, V. J. (2007). Incompetencia motriez e ideología del rendimiento en educación física: el caso de un alumno con discapacidad intelectual. Motricidad. European Journal of Human Movement, 19, 165-190.

Pérez, J. (2009) La investigación en Actividad físicas y deportes adaptados: un camino por recorrer. Revista Internacional de Ciencias del Deporte, 16, 1-3.

Ruiz, L. M. (1995). Competencia motriz. Elementos para comprender el aprendizaje motor en Educación física Escolar. Madrid: Gymnos.

Savelsbergh, G. J. P., Van der Kamp, J., Williams, A. M., \& Ward, P. (2005). Anticipation and visual search behaviour in expert soccer goalkeepers. Ergonomics, 48, 1686-1697.

Van Biesen, D., Vereller, J., Meyer, C., Mactavish, J., Van de Vliet, P. \& Vanlandewijck, Y. (2010). The ability of elite table tennis players with intelectual disabilities to adapt their service/return. Adapted Physical Activity Quarterly, 27, 242-257.

Verdugo, M.A.(2003).Análisis dela definición de discapacidadintelectual de la Asociación Americana sobre Retraso Mental de 2002. Siglo Cero: Revista Española sobre Discapacidad Intelectual, 34(1), 519.

Whiting, H. T. (1986). Isn't there a catch in it somewhere? Journal of Motor Behavior, 18(4), 486-491.

Williams, A. M. (2000). Perceptual skill in soccer: Implications for talent identification and development. Journal of Sports Sciences, $18,737-750$.

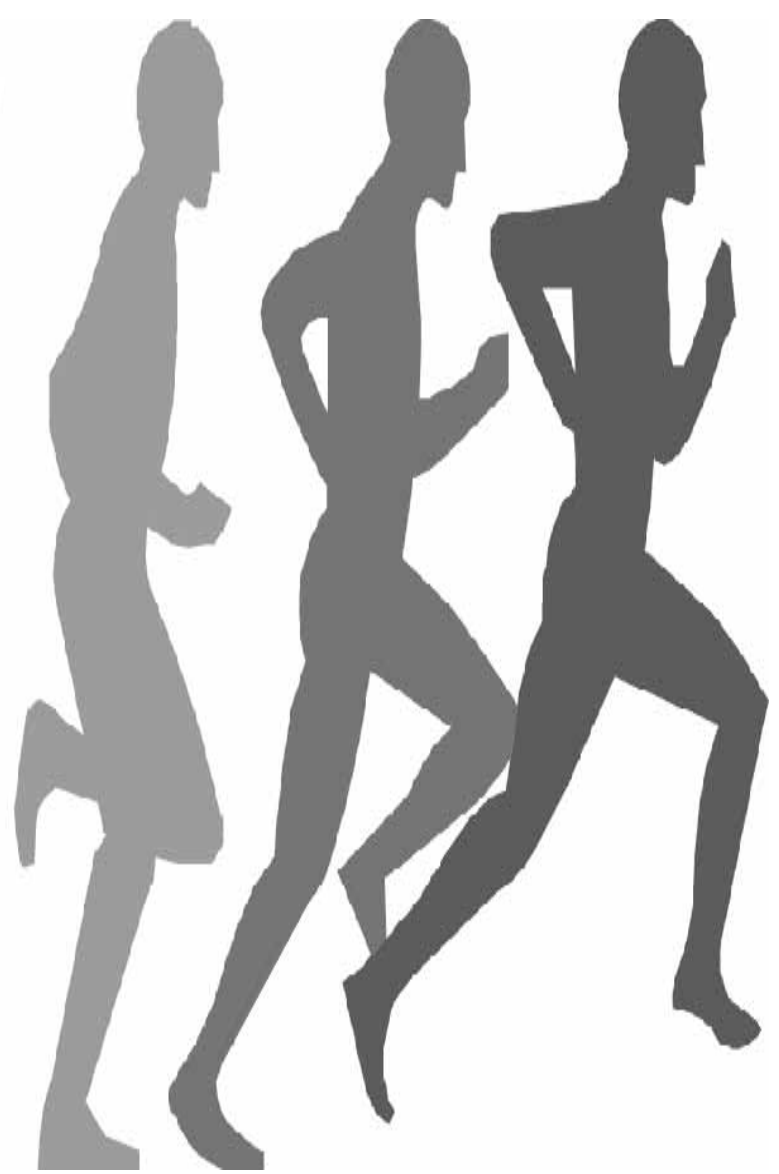

